\title{
More Is Less: A Disinhibited Prefrontal Cortex Impairs Cognitive Flexibility
}

\author{
Aaron J. Gruber, ${ }^{1,3}$ Gwendolyn G. Calhoon, ${ }^{1}$ Igor Shusterman, ${ }^{1}$ Geoffrey Schoenbaum, ${ }^{1,2}$ Matthew R. Roesch, ${ }^{1,4}$ \\ and Patricio 0'Donnell ${ }^{1,2}$ \\ Departments of ${ }^{1}$ Anatomy and Neurobiology, and ${ }^{2}$ Psychiatry, University of Maryland School of Medicine, Baltimore, Maryland 21201, ${ }^{3}$ Department of \\ Neuroscience, University of Lethbridge, Lethbridge, Alberta T1K 3M4, Canada, and ${ }^{4}$ Department of Psychology and Program in Neuroscience and Cognitive \\ Science, University of Maryland College Park, College Park, Maryland 20742
}

The prefrontal cortex (PFC) is critical for decision making, and it becomes dysfunctional in many neuropsychiatric disorders. Studies in schizophrenia patients and relevant animal models suggest loss of PFC inhibitory interneuron function. For instance, rats with a neonatal ventral hippocampal lesion (NVHL) show a deficient modulation of PFC interneurons by dopamine (DA). Whether the PFC becomes disinhibited in this model and alters decision making remains to be determined. Here, we recorded neural activity in the medial PFC of NVHL rats during a reward-discounting choice task that activated DA systems. Rats were trained to sample odors that instructed them to select one of two feeders that delivered unequal amounts of liquid. Putative pyramidal neurons in the PFC were hyperactive whereas task-related field potential oscillations were significantly reduced in NVHL rats, consistent with impaired interneuron activation by DA during odor sampling leading to disorganized processing. Cognitive flexibility was tested by examining response bias and errors after reversing reward outcomes. NVHL rats demonstrated impaired flexibility as they were less able to track changes in reward outcome and made more response errors than controls did. Reducing cortical excitability with the metabotropic glutamate receptor $2 / 3$ agonist LY379268 (1 mg/kg, i.p.) improved behavioral flexibility in NVHL rats but not controls. Furthermore, D2 dopamine receptors were involved, as the antagonist eticlopride $(0.02 \mathrm{mg} / \mathrm{kg}$, i.p. $)$ reduced the ability to switch only in control animals. We conclude that NVHL rats present PFC disinhibition, which affects neural information processing and the selection of appropriate behavioral responses.

\section{Introduction}

The prefrontal cortex (PFC) is a central component of current hypotheses on schizophrenia pathophysiology (Harrison and Weinberger, 2005; Lewis et al., 2005). This brain region is essential for flexible decision making, particularly when it involves response conflicts, effort, and delays (Ragozzino et al., 1999; Manes et al., 2002; Rushworth and Behrens, 2008). PFC deficits in schizophrenia include altered working memory (Carter et al., 1998) and perseverance (Fey, 1951) and a decline in cognitive flexibility and reward processing (Gold et al., 2008). Postmortem studies consistently reveal loss of markers associated with GABA interneurons, such as parvalbumin, in the PFC and other cortical regions in brains from schizophrenia patients (Benes and Berretta, 2001; Lewis et al., 2005). Further evidence of interneuron dysfunction comes from abnormal frontal lobe high-frequency electroencephalographic oscillations in schizophrenia (Spencer et al., 2003) that are known to reflect interneuron activity (Szabadics et

Received Sept. 2, 2010; revised 0ct. 4, 2010; accepted 0ct. 21, 2010.

This work was supported by grants from the National Institutes of Health (MH57683 to P.0.; R01-DA015718 and R01-AG027097 to G.S.; K01-DA021609 to M.R.R.) and the Natural Sciences and Engineering Research Council and Alberta Heritage Foundation for Medical Research (to A.J.G.). LY379268 was a generous donation from Eli Lilly.

Correspondence should be addressed to Dr. Patricio O'Donnell, University of Maryland School of Medicine, Program in Neuroscience, Department of Anatomy and Neurobiology, 20 Penn Street, s251, Baltimore, MD 21201. E-mail: podon002@umaryland.edu.

DOI:10.1523/JNEUROSCI.4623-10.2010

Copyright $\odot 2010$ the authors $\quad 0270-6474 / 10 / 3017102-09 \$ 15.00 / 0$ al., 2001). Therefore, reproducing interneuron deficits in animals provides a tool to test the behavioral impact of PFC disinhibition.

Abnormal interneurons are characteristic of several rodent models of schizophrenia. Reduced parvalbumin levels have been observed in the PFC of rats treated with the antimitotic MAM (methylazoxymethanol acetate) (Lodge et al., 2009), NMDA antagonists (Behrens et al., 2007), and in transgenic mice with glutathione deficits (Cabungcal et al., 2006) or a dominant-negative DISC1 gene (Hikida et al., 2007). Furthermore, a neonatal ventral hippocampal lesion (NVHL) alters the postnatal maturation of PFC circuits (O'Donnell et al., 2002; Tseng et al., 2007; Tseng et al., 2008) by interfering with cortical development at a critical period (Lipska, 2004). Adult NVHL rats have pyramidal neurons with abnormal dendritic morphology (Flores et al., 2005) and reduced levels of the GABA-synthesizing enzyme GAD67 (Lipska et al., 2003). Medial PFC pyramidal neurons of NVHL rats recorded in vivo respond to stimulation of the ventral tegmental area (VTA), the origin of dopamine (DA) projections to the PFC, with an increase in firing (O'Donnell et al., 2002) instead of the normal decrease (Lewis and O'Donnell, 2000). This aberrant response reflects the inability of $\mathrm{D}_{2} \mathrm{DA}$ agonists to activate interneurons in adult rats with a NVHL (Tseng et al., 2008). These data suggest that a DA-mediated disinhibition of prefrontal output may be a central feature of rodent models that recapitulate schizophrenia deficits. Therefore, these models provide an opportunity to assess whether the disinhibition proposed to exist in schizophrenia affects PFC-dependent behaviors. The NVHL 

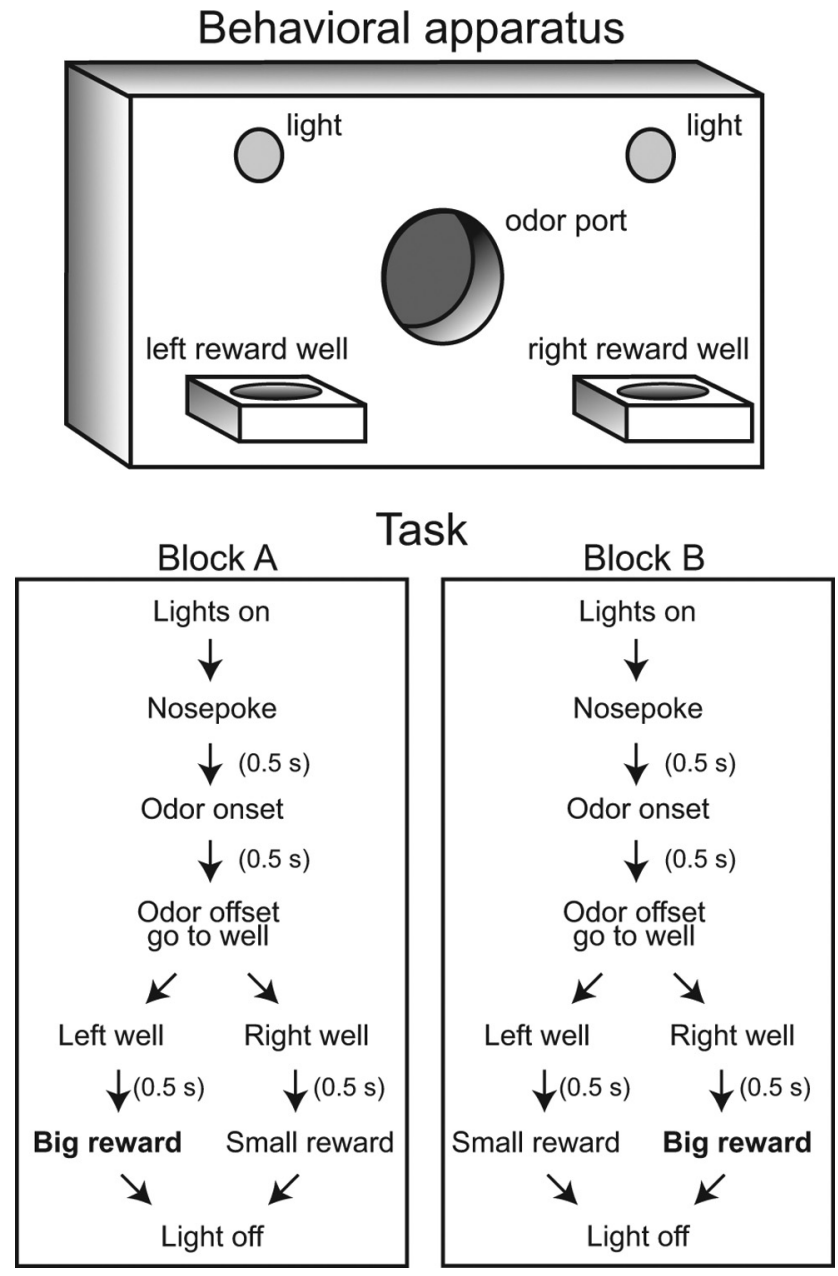

Figure 1. Size-discounting task. Sketch of task apparatus (top) and time course (bottom) of task events for the two blocks used during recordings sessions. model in particular is suitable to address schizophrenia-related cognitive deficits, as it reproduces a wide array of behavioral phenomena, including working memory deficits (Lipska et al., 2002). To directly test whether NVHL rats exhibit PFC disinhibition and whether this condition is responsible for impaired cognition, we recorded neural activity in the medial PFC of adult rats that had received a NVHL or a sham operation during a value-based choice task that activates DA neurons (Roesch et al., 2007). We expected that disinhibition would be evident in NVHL animals during cue-evoked DA release because of the deficient activation of PFC interneurons by DA and reducing glutamate release would improve performance.

\section{Materials and Methods}

Subjects, surgeries, and histology. All experiments were performed according to United States Public Health Service Guide for the Care and Use of Laboratory Animals and were approved by the University of Maryland Institutional Animal Care and Use Committee. Procedures for neonatal lesions, training, and data collection follow previously described methods (Lipska et al., 1993; Roesch et al., 2006). On postnatal day (P) 7-8, male Long-Evans pups born onsite were bilaterally injected with $0.3 \mu \mathrm{l}$ of either artificial CSF (sham) or ibotenic acid $(0.3 \mu \mathrm{g}$; NVHL) in the ventral hippocampus ( $3 \mathrm{~mm}$ posterior, $\pm 3.5 \mathrm{~mm}$ lateral, and $5 \mathrm{~mm}$ ventral to bregma). A total of $15 \mathrm{NVHL}$ and 14 sham rats were used in this study. Rats were group housed until the start of behavioral training at P41. After two sessions of acclimation to the behavioral boxes, rats were water restricted and trained on the two-odor discrimination portion of the task for equal amounts of liquid sucrose reward from the left and right delivery wells. Once rats had acquired the task at $>80 \%$ accuracy, ad libitum access was given to water for $4 \mathrm{~d}$ and drivable electrode bundles of eight 25- $\mu$ m-diameter FeNiCr wires (Stablohm 675; California Fine Wire) were implanted in the left prelimbic cortex $(3.3 \mathrm{~mm}$ anterior, $+0.6 \mathrm{~mm}$ lateral, and 2.8 to $4.5 \mathrm{~mm}$ ventral to bregma) using aseptic techniques and under isoflurane anesthesia. Immediately before implantation, wires were freshly cut and electroplated with platinum $\left(\mathrm{H}_{2} \mathrm{PtCl}_{6}\right.$; SigmaAldrich) to an impedance of $\sim 300 \mathrm{k} \Omega$. Following $10-15 \mathrm{~d}$ of recovery, rats were water-restricted and brought to the lab for daily recording sessions from P99-P142. Electrodes were advanced at the end of each session. Following 6 weeks of recording sessions, rats were killed and brains were fixed in $4 \%$ paraformaldehyde for $24-48 \mathrm{~h}$ and then saturated with $30 \%$ sucrose solution for cryoprotection. Brain slices were cut on a freezing microtome, mounted on gelatin-coated slides, and Nissl-stained to allow for histological verification of hippocampal structure and recording electrode placement. Only rats with successful NVHL or sham procedures were included in the analyses. In addition, only electrophysiological data from rats with properly placed electrode tracks in the prelimbic cortex were analyzed.

Behavioral training. Training and recordings were conducted in aluminum chambers $\sim 0.5$ $\mathrm{m}$ long on each side. The task apparatus consisted of two liquid-delivery feeder wells on either side of a centrally located odor port, with lights located above the wells (Fig. 1). The odor port was connected to an air flow dilution olfactometer to allow rapid delivery of olfactory cues, and the task was controlled with a computer. Three easily discriminable odors (verbena oliffac, cedryl acetyl trubek, camekol DH) where chosen from compounds obtained from International Flavors and Fragrances. Rats were initially trained on a two-odor discrimination task in which one of two odors were presented with equal probability at each trial. One odor indicated liquid sucrose availability at the right well and the other odor indicated
Figure 2. NVHL rats can learn the task. $\boldsymbol{A}, \boldsymbol{B}$, Number of trials $(\boldsymbol{A})$ and number of response errors $(\boldsymbol{B})$ for NVHL (red) and sham (black) rats during the initial training sessions in which only the two forced-choice trials were presented. Delays between task events were increased across trials to the values used in the full task during recording sessions. There were no differences in the number of trials and response errors between groups (ANOVA, $p>0.05, n=11$ ). C, Illustration of histologically verified damage to the ventral hippocampus in adult NVHL rats indicated on a standard atlas (Paxinos and Watson, 2005). Gray and dark regions show the largest and smallest extent, respectively, of damage across rats. 
reward availability in the left well. For this phase of training, reward amounts were equal for both wells and task delays were gradually increased across sessions. During postsurgical recording sessions, rats performed a modified version of the odor discrimination task in which an additional novel odor was presented that allowed for selection of either reward well for liquid sucrose, and the amount of reward was always unequal for the two wells. Before each session, one well was pseudorandomly chosen to deliver large rewards (double the amount of the small reward) for the first half of the session, and subsequently gave small rewards for the second half of the session. The serial order of odor presentation was generated such that each odor had a $1 / 3$ probability of being presented on any trial, but no single odor was presented in more than three consecutive trials. Blocks consisted of at least 60 trials and continued until rats demonstrated a bias away from the discounted well, defined as at most four responses to this well over the previous 10 free choice trials.

Pharmacological treatments. Separate groups of NVHL $(n=4)$ and control $(n=4)$ rats were well trained (27 sessions starting at P40) and given saline or $1 \mathrm{mg} / \mathrm{kg}$ intraperitoneally of the metabotropic glutamate receptor agonist $2 / 3$ LY379268 (Eli Lilly) in saline before behavioral testing at $\mathrm{P} 125$, to decrease glutamate release. The $\mathrm{pH}$ of vehicle and drug was adjusted to $5-6$, and the amount injected was $\sim 0.1 \mathrm{ml}$ (depending on rat's weight). Rats performed the task starting 30-35 min after injection. We modified the block structure of the task to reduce variability of behavior across sessions and improve detection of drug effects with fewer sessions. We first determined the rat's rewardwell preference by allowing 20 trials with equal reward outcomes. The reward size was then doubled for the nonpreferred well on the first block of trials (trial number 21-90), and the reward contingencies were reversed for the second block of trials (trial number 91-180). Groups of naive $(n=5)$ and NVHL $(n=5)$ rats were similarly trained and then tested using the same procedure following administration of the $\mathrm{D}_{2}$ antagonist eticlopride. These rats were given $\sim 0.1 \mathrm{ml}$ intraperitoneally of either vehicle or eticlopride $(0.02 \mathrm{mg} / \mathrm{kg}) 30-35 \mathrm{~min}$ before task initiation.

Electrophysiological recordings. Procedures were the same as described previously (Roesch et al., 2007). Amplification and recording of neural activity were conducted using Plexon Multichannel Acquisition Processor Systems. Gains and thresholds for single unit recordings were set for each channel before each session. All threshold crossings were recorded, along with $800 \mu$ s waveform segments for subsequent analysis. Simultaneous with single unit data collection, field potentials from two to three microwires were bandpass filtered $(0.77-300 \mathrm{~Hz})$, amplified $(5000 \times)$, sampled $(1 \mathrm{kHz})$, and recorded to a computer hard drive. The electrode array was advanced $40-80 \mu \mathrm{m}$ after each session.

Analysis. Units were sorted using a template-matching algorithm implemented in Offline Sorter software from Plexon. Files containing sorted units, field potentials, and timestamps of task events were processed using custom routines and the Chronux toolbox (http://chronux.org) implemented in Matlab. For single unit responses, regions of significant.
C
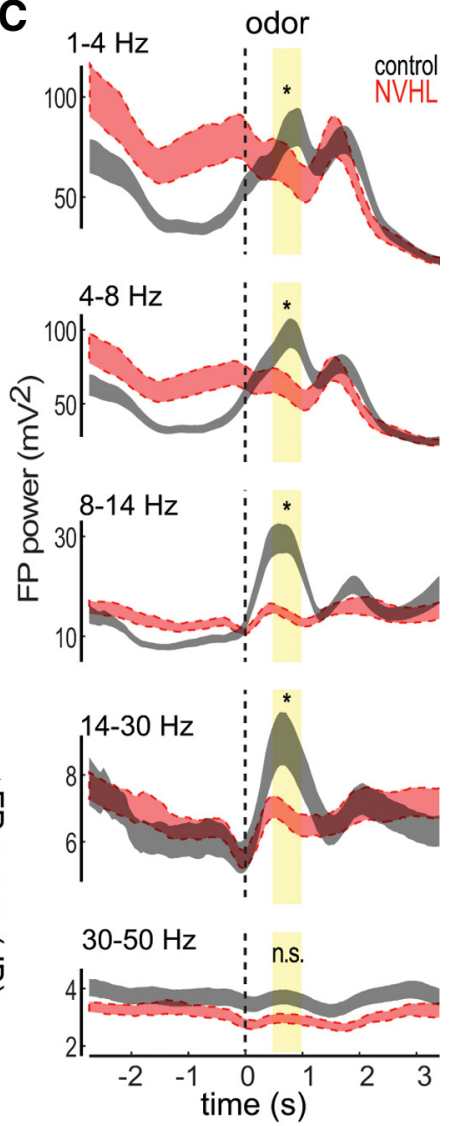

odor sampling

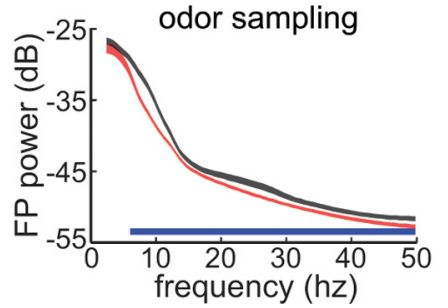

Loss of field-potential modulation in NVHL rats during the task. $A$, Histologically identified electrode tracks in the medial PFC for control and NVHL rats. Prelimbic (PL) and infralimbic (IL) cortical areas are labeled according to a standard atlas (Paxinos and Watson, 2005). B, Task-averaged time-frequency spectrograms of field potentials reveal reduced power for NVHL rats tom; $n=6$ ) and sham controls (top; $n=5$ ). Data are aligned to odor port entry, normalized to the 0.5 s interval before port entry, and include only field potentials recorded during correct trials. C, Loss of field potential (FP) power in NVHL rats is observed cross several frequency bands. Summed field potential power within conventional frequency bands for NVHL (red) and contro indicated by *) for all frequency bands $<30 \mathrm{~Hz}$. D, Field potential power spectra for NVHL (red) and control (gray) rats during baseline and odor presentation epochs, revealing less oscillation amplitude in NVHL rats in gamma and other frequency ranges ( $p<0.05$ by jackknifed U-statistic indicated by a blue line below the plot). Shaded regions represent mean \pm SEM. n.S., Not

interest were defined relative to baseline ( 2 to $1 \mathrm{~s}$ before odor poke), odor presentation ( 0 to $0.5 \mathrm{~s}$ after odor onset), odor port exit ( 0.5 to $0 \mathrm{~s}$ before odor port exit), reward ( 0 to $1 \mathrm{~s}$ after reward delivery), and well exit ( 0 to $1 \mathrm{~s}$ after well exit). Changes in firing rate during the task were assessed by Wilcoxon test of the time-normalized number of spikes in each region of interest with respect to baseline for all correct trials. We then constructed group-averaged firing rates for each epoch using only those units that had a significant increase in rate. Group-averaged rates were compared by pairwise $t$ test of $z$-transformed rates during the region of interest. Two-tailed versions of all statistical tests were used. For analysis of field potentials, all trials in which the signal was clipped by reaching the upper or lower bounds were eliminated so as to avoid contamination by artifacts. We then computed time-frequency spectrograms using a multitaper method to segment field-potential signals into overlapping $0.5 \mathrm{~s}$ 

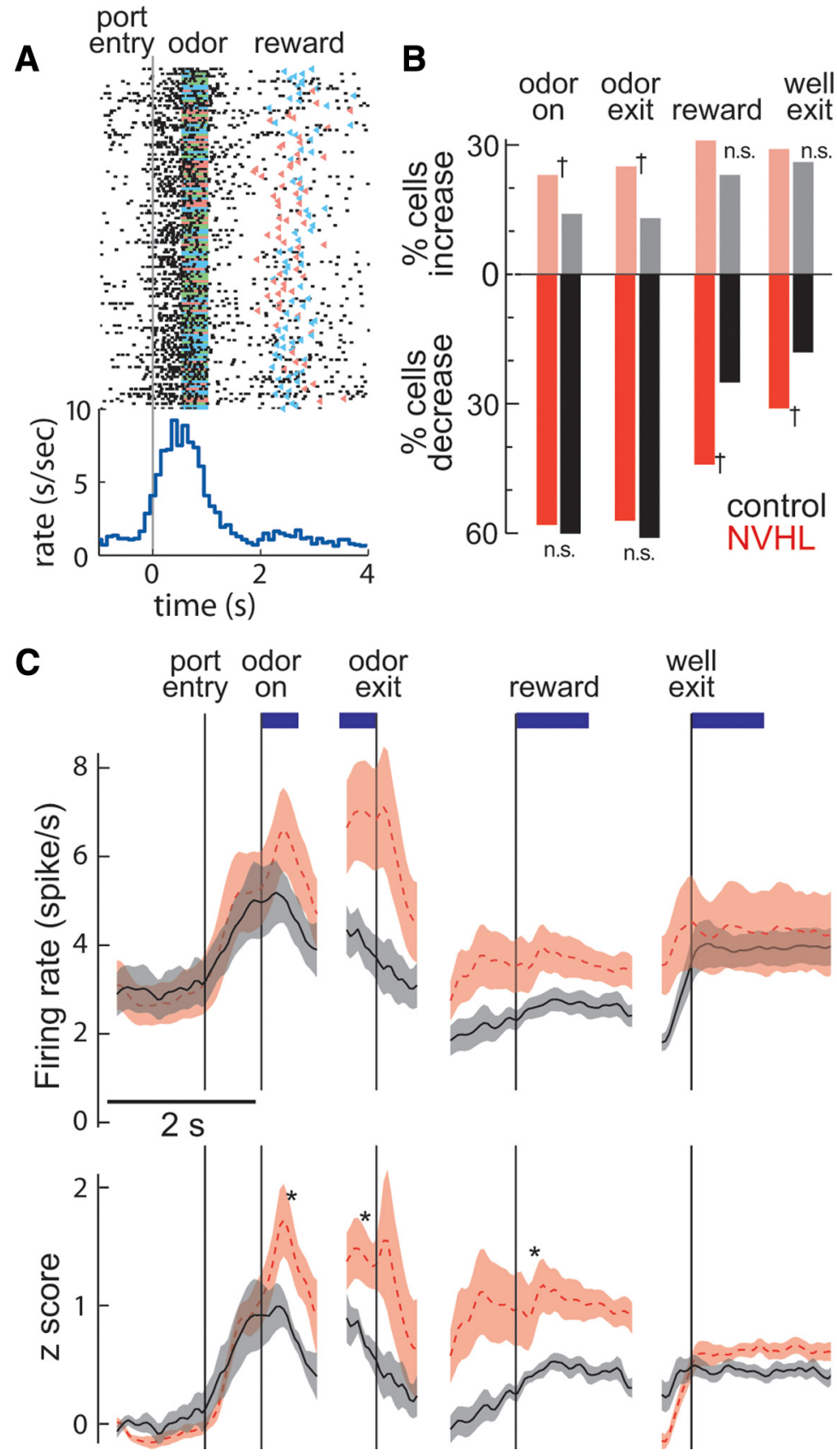

Figure 4. Single-unit responses during the task. $\boldsymbol{A}$, Raster plot and perievent time histogram for a putative pyramidal neuron recorded from a control rat performing the decision-making task. Colored bars indicate odor cue (green, free choice; red, forced right; blue, forced left) and triangles indicate reward delivery (red, right; blue, left). $\boldsymbol{B}$, Bar plots showing the percentage of units from control (black; $n=86$ ) and NVHL (red; $n=159$ ) rats responding with significant increases of firing during each region of interest labeled above the bars. †Differences between the number of units, computed by $\chi^{2}$. C, Group averages of raw firing rates (top) and zscores (bottom) for PFC single units that showed a significant increase in activity during task epochs for NVHL (dashed red line) and control (solid black line) rats. Data for each epoch are aligned to the task event indicated by labels above vertical lines. Baseline firing was not different between the two groups; however, NVHL rats showed increased firing during the task. ${ }^{*} p<0.05$ by $t$ test of mean cell firing during regions of interest marked by blue bars above plots. Lines indicate mean and shaded regions represent \pm SEM.

windows that were incremented $0.05 \mathrm{~s}$ for each successive window. Although two to three field channels were recorded simultaneously, only one channel per session was used for analysis in order to minimize oversampling of potentially dependent signals. The fast Fourier transform of each window segment was used to compute power as a function of frequency. Total power within conventionally defined frequency ranges was computed by integrating power over these ranges for each window. The
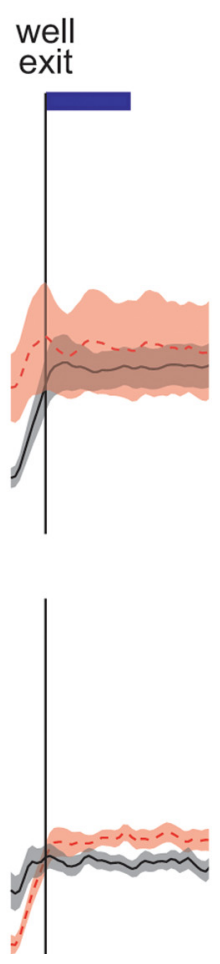

modulation of field potentials during odor presentation was computed by dividing the average power during odor presentation by the average power for an equal amount of time $(0.5$ s) before the odor port entry. This ratio was used as a regressor for the error rate on forcedchoice trials for each rat. Statistical testing of spectral densities was performed by a jackknifing U-statistic method (Arvesen, 1969), as implemented in the Chronux toolbox. Smoothing by convolution of data series with a Gaussian kernel was used for population firing-rate data $(\sigma=50 \mathrm{~ms})$ and for the session data comprising the plot of behavioral actions on freechoice trials ( $\sigma=2$ trials). All statistical tests were performed on raw (unsmoothed) data.

\section{Results}

\section{Value-based decision-making task}

Adult rats with a NVHL or sham-operated controls were trained to perform a choice task. Rats were trained to select one of two adjacent liquid delivery wells based on a brief olfactory cue presented in a centrally located odor port (Fig. 1). On each trial, one of three odors was presented in a pseudorandom order that (1) forced selection of only the right well, (2) forced selection of only the left well, or (3) allowed free selection of either well for liquid sucrose reward given after a constant delay. Behavior was initially shaped over 15 sessions using the two forced-choice odors for equal amounts of sucrose in both wells, and both groups achieved better than $80 \%$ accuracy. No differences were found in the number of trials performed or number of errors committed between groups, indicating normal odor sensitivity, motivation, and ability to learn stimulus-response associations (Fig. 2A,B). Subsequent sessions were organized into two trial blocks that were identical in all respects except for the amount of sucrose given, which was twice as large for the left well during one block and twice as large for the right during the other. These sessions included both forced- and free-choice trials presented in a pseudorandom order (60-100 trials per block). The block order was pseudorandomly chosen for each rat and session. Drivable electrode arrays were implanted in the prelimbic region of the medial PFC, and neural activity and behavioral actions were recorded for up to 25 sessions of the full task (free- and forced-choice trials with asymmetric rewards) following recovery from surgery. Infrared sensors were used to ensure the animals maintained head fixation in the odor port during the tasks. Video images of the rats performing the tasks were also used to ensure there were no obvious postural idiosyncrasies that could confound results. Only data from rats with histologically verified hippocampal structure that indicated successful sham (five rats) or NVHL (six rats) (Fig. 2C) procedures were 
Table 1. Number of neurons that increased, decreased, or did not change their rate during behaviorally relevant task epochs

\begin{tabular}{|c|c|c|c|c|}
\hline & Odor delivery & Exit odor port & Reward well & Exit reward well \\
\hline $\begin{array}{l}\text { NVHL ( } n=156) \text { (Increased, decreased, } \\
\text { did not change) }\end{array}$ & $37, * 93,26$ & $40,{ }^{*} 91,25$ & $49,70,{ }^{*} 37^{*}$ & $46,49,{ }^{*} 61^{*}$ \\
\hline $\begin{array}{l}\text { Control ( } n=87 \text { ) (Increased, decreased, } \\
\text { did not change) }\end{array}$ & $12,52,23$ & $11,53,23$ & $20,22,45$ & $23,16,48$ \\
\hline
\end{tabular}

*Significant differences of between-group frequencies $\left(\chi^{2} ; p<0.05\right)$.

included in subsequent analyses. No explicit cues about blocks were presented, so rats had to use the reinforcement history to discern the location of the large reward, which switched sides once during each session.

\section{Interneuron-dependent field-potential oscillations are impaired in NVHL rats during the task}

To assess interneuron function, we quantified interneurondependent oscillations in PFC local field potentials (LFPs). Highfrequency $(20-80 \mathrm{~Hz})$ cortical oscillations are the result of pyramidal cell-interneuron interactions (Traub et al., 2001). For instance, optogenetic stimulation of parvalbumin interneurons can drive cortical oscillations in that range (Cardin et al., 2009), indicating a strong role of interneuron activity in their genesis. Recordings were made from wires advanced through the prelimbic PFC region (Fig. $3 A$ ) as rats performed the task. The Fourier transform of windowed event-triggered LFPs was used to compute spectral composition of field potential oscillations as a function of time. We found broad-spectrum modulation of LFPs during the exposure to cues in control rats, but such modulation was attenuated in NVHL rats (Fig. $3 B$ ). In particular, the large increase in field-potential power during odor sampling was significantly reduced in NVHL rats for all frequency bands $<30 \mathrm{~Hz}$ (Fig. 3C) ( $t$ tests with Bonferroni correction; $\mathrm{df}=93$, all $p<1 \times$ $\left.10^{-8}\right)$. This difference of modulation did not stem from a general tonic reduction of field-potential fluctuations in NVHL rats, as the power of all frequency bands before the task initiation were not lower in these rats relative to controls. Thus, delta, theta, and beta components were enhanced in control rats during odor sampling, a period in which DA neurons become activated (Roesch et al., 2007). The increased beta component in control rats may reflect activation of interneurons, as this frequency range is normally associated with synchronized activation of fast-spiking interneurons (Szabadics et al., 2001). In NVHL rats, the increase in beta frequency was not present, suggesting that interneurons may not be recruited effectively during the decision-making epoch of the task. Interestingly, mean gamma band power was not significantly modulated during odor presentation in either group. However, recording electrodes were in deep cortical layers, which generate much less gamma-band power than superficial PFC layers (Roopun et al., 2006) and may thus require a more sensitive analysis. We therefore used a fine-grained analysis to directly compare the full power spectral density between groups (Fig. $3 D)$. NVHL rats had significantly less field-potential power in much of the gamma range during odor presentation and during baseline, suggesting a persistent deficit in interneurondependent oscillatory activity.

\section{Excessive cell firing during the task in NVHL rats}

A state of PFC disinhibition would yield excessive firing and/or an abnormally high number of pyramidal neurons recruited during the task. As interneuron deficits in NVHL animals are exacerbated by DA (Tseng and O'Donnell, 2007; Tseng et al., 2008), enhanced pyramidal-cell firing should become evident during

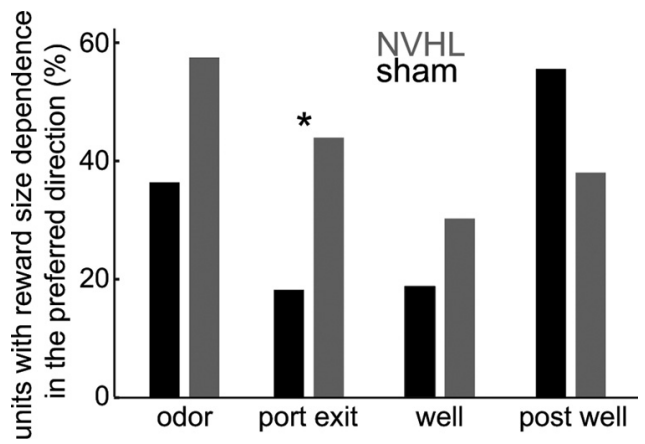

Figure 5. Bar plot showing the proportion of units that had a dependence on reward size for sham (black) and NVHL (gray) rats over multiple task epochs. Reward dependence was assessed by computing firing in each epoch for large and small reward trials to the preferred response direction for each unit, and testing for significant differences of firing in these conditions using the Wilcoxon rank sum test. ${ }^{*} p<0.05$ by $\chi^{2}$ test between groups for each epoch.

odor presentation, when DA neurons in the VTA are activated (Roesch et al., 2007). To address this possibility, we examined electrical activity of individual neurons recorded from the medial PFC using the same wires as the field-potential data (87 neurons from five sham rats, and 159 neurons from six NVHL rats). In both groups, some of these putative pyramidal neurons increased their firing rate during some portion of the task relative to the pretrial baseline (Fig. 4A). However, more units increased their activity during the odor presentation in NVHL rats than in controls $\left(p<0.05, \chi^{2}\right)$ (Fig. $4 B$; Table 1). Moreover, the average increase in firing during the task was higher in NVHL rats ( $t$ tests comparing increases in the subset of neurons that increased cell firing; $\mathrm{df}=67 ; p<0.05$ ) (Fig. $4 C$ ). The largest difference in firing occurred when the rats were at the odor port before movement; rate increases from baseline were $97 \%$ for NVHL rats and $65 \%$ for controls. Increased action potential firing in NVHL rats was specific to task periods from odor presentation to reward delivery, when phasic firing in DA neurons is observed; differences in firing between NVHL and control rats were not observed before or after the task. Since DA neuron responses are larger for cues that indicate larger rewards (Roesch et al., 2007), cues associated with large rewards should induce higher responses in a greater number of PFC pyramidal neurons in NVHL rats. To assess the reward dependence of firing, we controlled for directional selectivity by only analyzing trials in the preferred direction of each neuron. Compared with shams, more pyramidal neurons in NVHL rats showed increases in firing for higher reward during the $1 \mathrm{~s}$ period following odor-delivery onset $\left(p=0.03, \chi^{2}\right)$ (Fig. $5)$. This overall pattern of results is consistent with the proposal that the loss of inhibitory mechanisms within the medial PFC in NVHL rats results in excessive and presumably inappropriate firing in task-related units, and that this process is exacerbated in conditions of high dopamine.

Differences were also observed during and immediately after reward delivery. In these task epochs, more PFC neurons decreased firing in NVHL rats than in controls $\left(p<0.05, \chi^{2}\right)$ (Fig. 
$4 B$; Table 1). The group-averaged firing rates of these neurons, however, were not different between groups and did not discriminate reward size, choice, or error trials (data not shown), and were therefore not analyzed further. Thus, the higher proportion of PFC neurons inhibited during reward delivery in NVHL rats may relate to some aspects of reward processing, but is unlikely to contribute to differences in decision making in this task.

\section{Cognitive flexibility is impaired in NVHL rats}

Both NVHL and control rats were able to track changes in the location of the large reward across trials. Rats learned to select the well associated with the large reward in free-choice trial blocks and also exhibited faster and more accurate responding to the well associated with the large reward on forced-choice trials as each block progressed. However, NVHL rats showed less flexibility in their choices than controls did. Preference for the bigger reward on free-choice trials developed more slowly in NVHL than in sham rats (lesion status by trial interaction in a repeatedmeasures ANOVA, $F_{(1,4290)}=10.0, p<0.01$ ) (Fig. 6A). NVHL rats made more errors on forced-choice trials for both small and large rewards than controls (ANOVA, $F_{(1,187)}=40.2, p<0.001$; Tukey-Kramer post hoc analysis; $p<0.05$ ) (Fig. $6 B$ ). These deficits were associated with shorter reaction times than controls (ANOVA, $F_{(1,187)}=4.6, p<0.05$; Tukey-Kramer post hoc analysis; $p<0.05$ ) (Fig. $6 C$ ) and a nonsignificant trend to exit the odor port prematurely ( $t$ test; $\mathrm{df}=93, p=0.19$ ) (Fig. $6 D$ ), suggesting that NVHL rats behaved impulsively. As NVHL rats showed less bias than shams in free-choice trials as blocks progressed, they may be expected to show faster reversal when reward contingencies were changed. However, we found that NVHL rats changed their well preference over the first five freechoice trials following the transition to the second block to a lower extent than control rats ( $t$ test, $\mathrm{df}=93, p<0.05$ ) (Fig. $6 E$ ), suggesting the possibility of perseverative errors. Thus, NVHL rats were able to discern and act on differences in reward outcome, but did so less efficiently than controls.

To explore the functional significance of the local fieldpotential modulation during odor sampling, we examined the relationship between increases of field-potential power during odor presentation and performance on forced-choice trials in each control rat. These variables were correlated across the frequency band that showed modulation during the odor sampling $(1-30 \mathrm{~Hz})$, which revealed a linear relationship $\left(r^{2}=0.8 ; p<\right.$ 0.02 ) (Fig. $6 F$ ), indicating that modulation of field-potential oscillations covaried with task performance. This observation suggests that reduced prelimbic PFC synchronization contributes to the poorer response selection in NVHL rats.

\section{Reducing glutamate transmission improves behavior of NVHL rats}

To determine whether impaired decision making in NVHL rats is indeed due to a disinhibited PFC, we tested whether reducing glutamate release in these animals ameliorated behavioral deficits. Task performance was assessed following systemic administration of a metabotropic glutamate receptor $2 / 3$ (mGluR2/3) agonist (LY379268; $1 \mathrm{mg} / \mathrm{kg}$ i.p.) in a separate group of rats well trained on a modified version of the task with shorter blocks and two response reversals (Fig. 7A). This class of agonists acts presynaptically to reduce glutamate release (Rorick-Kehn et al., 2007) and has been shown to reduce excessive medial PFC pyramidal neuron firing in the NMDA antagonist model of schizophrenia (Homayoun et al., 2005). Compared with vehicle, drug administration in NVHL rats did not affect reaction times but
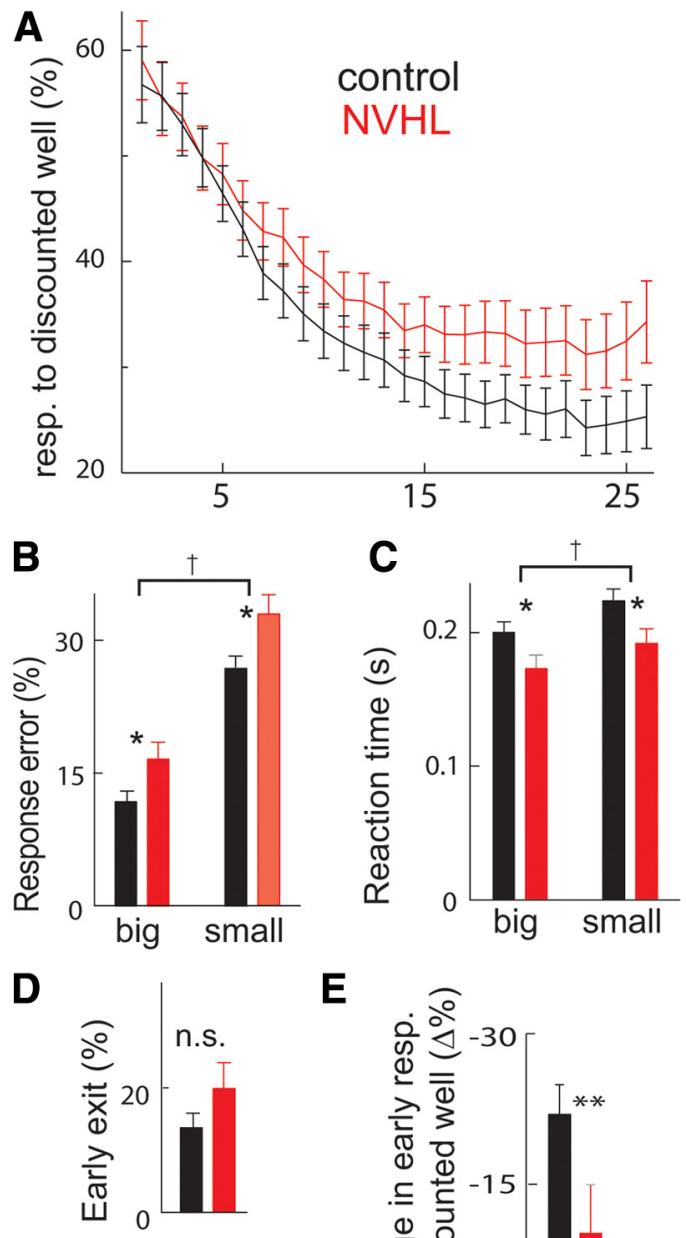

E
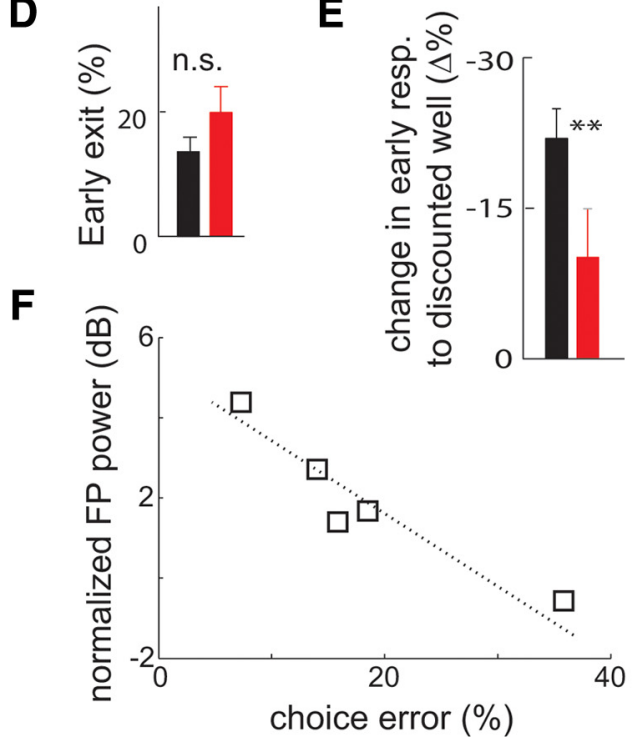

Figure 6. Behavioral deficits in NVHL rats. $\boldsymbol{A}$, Plot of group-averaged response (resp.) choices during sequential free-choice trials within behavioral blocks, showing that both groups of rats develop a bias against the well giving small rewards as blocks progress, but NVHL rats (gray, $n=6$ ) showed less bias than control rats (black, $n=5$ ). $\boldsymbol{B}$, Bar plot of response errors on forced-choice trials showing that rats made more response errors on forced trials for small rewards and that NVHL rats made more response errors than controls. C, Bar plot of reaction times showing that rats had faster reaction times for big rewards than small rewards, and NVHL rats had faster reaction times than controls for both reward sizes. $D$, Bar plot of premature exits from the odor port showing that NVHL rats had a nonstatistically significant trend to make more premature exits from the odor port. $\boldsymbol{E}$, Bar plot of mean change in responses away from the discounted reward well on the first five free-choice trials in each block compared with the preblock bias, showing response perseveration in NVHL rats on the second block compared with controls. For all plots, significance at the $95 \%$ level by main effects of two-way ANOVA ( $\dagger$ ), Tukey-Kramer post hoc test $\left(^{*}\right)$ and $t$ test $\left(^{* *}\right)$ are indicated. Error bars indicate \pm SEM. $\boldsymbol{F}$, Changes in field-potential power during odor presentation correlate with performance in the task. Error rate during forced-choice trails plotted against average power during odor presentation normalized by the preodor value for the $1-30 \mathrm{~Hz}$ frequency band in control rats. The line was generated by linear regression of data points. n.s., Not significant. 
increased responding for the well with higher reward on free-choice trials (drugby-group interaction in a repeatedmeasures ANOVA, $F_{(1,6)}=10.3, p<0.02$; post hoc paired $t$ test, $p=0.01, n=4$ ) (Fig. $7 B$ ), while also reducing errors to the well with less reward on forced-choice trials (drug-by-group interaction in a repeatedmeasures ANOVA, $F_{(1,6)}=10.0, p<0.02$; post hoc paired $t$ test; $p<0.05)$. In contrast, no effects were found in a control group of sham-operated rats on either free-choice bias or response errors on forced-choice trials $(n=4)$ (Fig. 7C). Thus, multiple aspects of task performance improve by reducing cortical excitability in NVHL rats.

\section{$D_{2}$ antagonists disrupt task performance} Interneuron deficits in the medial PFC of NVHL rats involve $\mathrm{D}_{2}$ dopamine receptors (Tseng et al., 2008). $D_{2}$ agonists increase interneuron excitability in naive and sham rats, but not in slices from NVHL rats. To test whether $\mathrm{D}_{2}$ receptor function is linked to behavioral performance, we administered a $\mathrm{D}_{2}$ antagonist (eticlopride, $0.02 \mathrm{mg} / \mathrm{kg}$, i.p.) to a separate group of rats trained on the task. Compared with vehicle, drug administration in naive rats impaired selection of the well delivering larger reward on free-choice trials (drug-by-group interaction in a repeated-measures ANOVA, $F_{(1,6)}=9.9, p<0.02$; post hoc paired $t$ test; $n=5 ; p<0.05)$ and showed a trend toward increased premature responding (drug-by-group interaction in a repeated-measures ANOVA, $F_{(1,6)}, p=$ 0.15 ) (Fig. 7D). This finding suggests correct performance on this decision-making task involves $\mathrm{D}_{2}$ DA receptors. As NVHL rats have intrinsic $\mathrm{D}_{2}$-related PFC deficits, we expected antagonism of this receptor type to have attenuated behavioral effects on NVHL compared with naive rats. Consistent with our hypothesis, administration of the same dose of $\mathrm{D}_{2}$ antagonist (eticlopride, $0.02 \mathrm{mg} / \mathrm{kg}$, i.p.) to a separate group of NVHL rats did not produce robust effects on behavior (Fig. 7E), providing further evidence supporting a $\mathrm{D}_{2}$ DA component of the decision-making deficits in these animals.

\section{Discussion}

We monitored behavioral actions and electrical activity in the prelimbic PFC while rats performed a choice task with asymmetric reward outcomes that varied in time. Although NVHL rats were able to track changes in reward outcomes by selecting the wells delivering larger rewards, their performance was less flexible than that of control rats. During recording sessions in which the location of the large reward varied across trial blocks, NVHL rats did not learn to choose the well giving large rewards as rapidly as control rats on free-choice trials and made more response errors on forced-choice trials. These behavioral deficits were associated with increased firing of medial PFC putative pyramidal neurons and a reduction in the power of PFC field potentials during odor sampling and ameliorated by pharmacological reduction of glutamate release. These data reveal alterations in both behavior and PFC physiology in adult rats with a NVHL that are tightly timed to epochs in which response decisions are made and support the idea that cortical hyperactivity impairs function.

The degraded performance of NVHL rats on the choice task is consistent with known cognitive deficits in this model. For example, the reduced selection of larger rewards on free-choice trials may reflect problems in forming or using information about the location of the well that gives large rewards. These problems could arise either from deficits in learning from past cue-response-reward outcomes or from deficits in using such information in working memory processes (Chambers et al., 1996; Lipska et al., 2002) to guide actions. However, learning or working memory deficits would not account for the pattern of errors on forcedchoice trials or the overall reduction in reaction times. These behavioral features may be more related to the inability to use sensory information to overcome a prepotent response and may reflect impulsivity in NVHL rats, although a proper test of impulsivity remains to be conducted in this model. The reduced flexibility observed here in NVHL rats could also be interpreted as an equivalent of perseveration, a PFC-dependent phenomenon 
commonly observed in schizophrenia and PFC lesions (Milner, 1963). Indeed, NVHL rats have been shown to exhibit perseverative behaviors in a set-shifting task (Brady, 2009). The medial PFC is, in fact, important for inhibiting actions based on sensory cues, as medial PFC lesions or inactivation in otherwise normal rats lead to premature responses (Narayanan and Laubach, 2006) and reduce the ability to resolve conflicts in sensory cues (Haddon and Killcross, 2006). Interestingly, NVHL rats were not deficient in their ability to learn cue-response associations during shaping of the behavior in which only forced-choice trials were given for equal reward size in both wells. Rather, the deficits emerged clearly only when free-choice trials and unequal reward outcomes were introduced, suggesting that neurophysiological changes in NVHL rats impact more strongly the cognitively demanding aspects of the task.

These behavioral deficits were closely associated with physiological anomalies in the medial PFC. Putative pyramidal neurons increased their firing to a greater extent during the odor sampling in NVHL rats than in controls. At the same time increased firing was observed, NVHL rats exhibited a dramatic failure to increase the strength of field-potential oscillations presumed to reflect the activity of local inhibitory interneurons. Although we cannot rule out changes in the activity of VTA DA neurons in NVHL rats being involved in the present results, our previous data suggest altered PFC responses to burst firing of DA neurons. For example, medial PFC pyramidal neurons show abnormal increases in firing following VTA stimulation in NVHL rats (O'Donnell et al., 2002), consistent with the proposed DA-mediated disinhibition in this rodent model. Furthermore, whole-cell recordings in prelimbic PFC slices revealed that pyramidal neurons are more excitable (Tseng et al., 2007), whereas fast-spiking interneurons are less excitable in adult rats with a NVHL (Tseng et al., 2008). $\mathrm{D}_{2}$ DA agonists increase excitability of fast-spiking interneurons in slices from adult naive or sham rats (Tseng and O'Donnell, 2007), but not from NVHL rats (Tseng et al., 2008). Therefore, burst firing in VTA DA neurons should recruit interneurons in sham rats, but do so weakly in NVHL rats, thereby exacerbating disinhibition. As the period in the task in which increased firing and loss of beta activation were observed corresponds to the time of activation of DA neurons in the VTA (Roesch et al., 2007), we suggest that the abnormal response to DA and loss of modulation of interneuron firing contribute to the excessive firing and recruitment of more units in NVHL rats. Our observations of increased task-related firing and reduced high-frequency fieldpotential oscillations are complementary and expected outcomes of inhibitory dysfunction in PFC because of the important roles of GABAergic interneurons for regulating and synchronizing firing in neocortex (Traub et al., 1996; Szabadics et al., 2001; Cardin et al., 2009). The most parsimonious mechanism to account for our observations is the deficient activation of PFC fast-spiking interneurons under conditions of high DA that has been revealed in our previous experiments with the NVHL model. Inappropriate activation of output from medial PFC is expected to interfere with normal performance on cognitively demanding tasks, such as the one used here. According to this model, the abnormal response of medial PFC to dopamine release in NVHL rats may prevent cortical circuits from filtering out irrelevant information, leading to poor decision making. The ability to improve task performance by pharmacologically reducing glutamate release in NVHL rats may be due to a reduced pyramidal cell excitability that compensates for impaired inhibition such that filtering of PFC output is improved.

Cortical disinhibition is increasingly recognized as a critical element in schizophrenia pathophysiology. Indeed, postmortem studies and neurophysiological assessments reveal loss of interneuron function and impaired PFC information processing in schizophrenia (Benes and Berretta, 2001). Our data suggest that such a loss may lead to an acute imbalance of excitation-inhibition when mesocortical DA pathways are activated, resulting in spurious information flow in cortical circuits and thus impairing decision-making processes. Such a state of exaggerated and inefficient activity has previously been proposed for schizophrenia (Callicott et al., 2000; Winterer et al., 2006). Given the well documented loss of specific interneuron populations in schizophrenia and the deficits in beta and gamma EEG oscillations, in particular when evoked by sensory stimuli (Spencer et al., 2008), we propose that excessive "noisy" information flow may occur in patients during critical decision-making instances due to disinhibition. Indeed, a recent study revealed that, despite intact reward-based learning, schizophrenia patients show a poor performance in using contextual information to guide decision making when outcome values are considered (Heerey et al., 2008). Although our study focused on the PFC, it is possible that interneuron deficits and cortical disinhibition constitute a global phenomenon in animal models of schizophrenia and perhaps in the human condition as well. A combination of current therapeutic approaches targeting DA receptors with novel approaches addressing disinhibition in cortical circuits would therefore be likely to provide a more efficacious treatment of schizophrenia.

\section{References}

Arvesen JN (1969) Jackknifing U-statistics. Ann Math Stat 40:2076-2100. Behrens MM, Ali SS, Dao DN, Lucero J, Shekhtman G, Quick KL, Dugan LL (2007) Ketamine-induced loss of phenotype of fast-spiking interneurons is mediated by NADPH-oxidase. Science 318:1645-1647.

Benes FM, Berretta S (2001) GABAergic interneurons: implications for understanding schizophrenia and bipolar disorder. Neuropsychopharmacology 25:1-27.

Brady AM (2009) Neonatal ventral hippocampal lesions disrupt set-shifting ability in adult rats. Behav Brain Res 205:294-298.

Cabungcal JH, Nicolas D, Kraftsik R, Cuénod M, Do KQ, Hornung JP (2006) Glutathione deficit during development induces anomalies in the rat anterior cingulate GABAergic neurons: relevance to schizophrenia. Neurobiol Dis 22:624-637.

Callicott JH, Bertolino A, Mattay VS, Langheim FJ, Duyn J, Coppola R, Goldberg TE, Weinberger DR (2000) Physiological dysfunction of the dorsolateral prefrontal cortex in schizophrenia revisited. Cereb Cortex 10:1078-1092.

Cardin JA, Carlén M, Meletis K, Knoblich U, Zhang F, Deisseroth K, Tsai LH, Moore CI (2009) Driving fast-spiking cells induces gamma rhythm and controls sensory responses. Nature 459:663-667.

Carter CS, Perlstein W, Ganguli R, Brar J, Mintun M, Cohen JD (1998) Functional hypofrontality and working memory dysfunction in schizophrenia. Am J Psychiatry 155:1285-1287.

Chambers RA, Moore J, McEvoy JP, Levin ED (1996) Cognitive effects of neonatal hippocampal lesions in a rat model of schizophrenia. Neuropsychopharmacology 15:587-594.

Fey ET (1951) The performance of young schizophrenics and young normals on the Wisconsin Card Sorting Test. J Consult Psychol 15:311-319.

Flores G, Alquicer G, Silva-Gómez AB, Zaldivar G, Stewart J, Quirion R, Srivastava LK (2005) Alterations in dendritic morphology of prefrontal cortical and nucleus accumbens neurons in post-pubertal rats after neonatal excitotoxic lesions of the ventral hippocampus. Neuroscience 133:463-470

Gold JM, Waltz JA, Prentice KJ, Morris SE, Heerey EA (2008) Reward processing in schizophrenia: a deficit in the representation of value. Schizophr Bull 34:835-847.

Haddon JE, Killcross S (2006) Prefrontal cortex lesions disrupt the contextual control of response conflict. J Neurosci 26:2933-2940.

Harrison PJ, Weinberger DR (2005) Schizophrenia genes, gene expression, and neuropathology: on the matter of their convergence. Mol Psychiatry $10: 40-68$. 
Heerey EA, Bell-Warren KR, Gold JM (2008) Decision-making impairments in the context of intact reward sensitivity in schizophrenia. Biol Psychiatry 64:62-69.

Hikida T, Jaaro-Peled H, Seshadri S, Oishi K, Hookway C, Kong S, Wu D, Xue R, Andradé M, Tankou S, Mori S, Gallagher M, Ishizuka K, Pletnikov M, Kida S, Sawa A (2007) Dominant-negative DISC1 transgenic mice display schizophrenia-associated phenotypes detected by measures translatable to humans. Proc Natl Acad Sci U S A 104:14501-14506.

Homayoun H, Jackson ME, Moghaddam B (2005) Activation of metabotropic glutamate $2 / 3$ receptors reverses the effects of NMDA receptor hypofunction on prefrontal cortex unit activity in awake rats. J Neurophysiol 93:1989-2001.

Lewis BL, O'Donnell P (2000) Ventral tegmental area afferents to the prefrontal cortex maintain membrane potential 'up' states in pyramidal neurons via $\mathrm{D}_{1}$ dopamine receptors. Cereb Cortex 10:1168-1175.

Lewis DA, Hashimoto T, Volk DW (2005) Cortical inhibitory neurons and schizophrenia. Nat Rev Neurosci 6:312-324.

Lipska BK (2004) Using animal models to test a neurodevelopmental hypothesis of schizophrenia. J Psychiatry Neurosci 29:282-286.

Lipska BK, Jaskiw GE, Weinberger DR (1993) Postpuberal emergence of hyperresponsiveness to stress and to amphetamine after neonatal excitotoxic hippocampal damage: a potential animal model of schizophrenia. Neuropsychopharmacology 9:67-75.

Lipska BK, Aultman JM, Verma A, Weinberger DR, Moghaddam B (2002) Neonatal damage of the ventral hippocampus impairs working memory in the rat. Neuropsychopharmacology 27:47-54.

Lipska BK, Lerman DN, Khaing ZZ, Weickert CS, Weinberger DR (2003) Gene expression in dopamine and GABA systems in an animal model of schizophrenia: effects of antipsychotic drugs. Eur J Neurosci 18:391-402.

Lodge DJ, Behrens MM, Grace AA (2009) A loss of parvalbumin-containing interneurons is associated with diminished oscillatory activity in an animal model of schizophrenia. J Neurosci 29:2344-2354.

Manes F, Sahakian B, Clark L, Rogers R, Antoun N, Aitken M, Robbins T (2002) Decision-making processes following damage to the prefrontal cortex. Brain 125:624-639.

Milner B (1963) Effects of different brain lesions on card sorting. Arch Neurol 9:90-100.

Narayanan NS, Laubach M (2006) Top-down control of motor cortex ensembles by dorsomedial prefrontal cortex. Neuron 52:921-931.

O’Donnell P, Lewis BL, Weinberger DR, Lipska BK (2002) Neonatal hippocampal damage alters electrophysiological properties of prefrontal cortical neurons in adult rats. Cereb Cortex 12:975-982.

Paxinos G, Watson C (2005) The rat brain in stereotaxic coordinates, 5th Ed. San Diego: Academic.

Ragozzino ME, Wilcox C, Raso M, Kesner RP (1999) Involvement of rodent prefrontal cortex subregions in strategy switching. Behav Neurosci 113:32-41.
Roesch MR, Taylor AR, Schoenbaum G (2006) Encoding of timediscounted rewards in orbitofrontal cortex is independent of value representation. Neuron 51:509-520.

Roesch MR, Calu DJ, Schoenbaum G (2007) Dopamine neurons encode the better option in rats deciding between differently delayed or sized rewards. Nat Neurosci 10:1615-1624.

Roopun AK, Middleton SJ, Cunningham MO, LeBeau FE, Bibbig A, Whittington MA, Traub RD (2006) A beta2-frequency $(20-30 \mathrm{~Hz})$ oscillation in nonsynaptic networks of somatosensory cortex. Proc Natl Acad Sci U S A 103:15646-15650.

Rorick-Kehn LM, Johnson BG, Burkey JL, Wright RA, Calligaro DO, Marek GJ, Nisenbaum ES, Catlow JT, Kingston AE, Giera DD, Herin MF, Monn JA, McKinzie DL, Schoepp DD (2007) Pharmacological and pharmacokinetic properties of a structurally novel, potent, and selective metabotropic glutamate $2 / 3$ receptor agonist: in vitro characterization of agonist (-)(1R,4S,5S,6S)-4-amino-2-sulfonylbicyclo[3.1.0]-hexane-4,6-dicarboxylic acid (LY404039). J Pharmacol Exp Ther 321:308-317.

Rushworth MF, Behrens TE (2008) Choice, uncertainty and value in prefrontal and cingulate cortex. Nat Neurosci 11:389-397.

Spencer KM, Nestor PG, Niznikiewicz MA, Salisbury DF, Shenton ME, McCarley RW (2003) Abnormal neural synchrony in schizophrenia. J Neurosci 23:7407-7411.

Spencer KM, Niznikiewicz MA, Shenton ME, McCarley RW (2008) Sensory-evoked gamma oscillations in chronic schizophrenia. Biol Psychiatry 63:744-747.

Szabadics J, Lorincz A, Tamás G (2001) Beta and gamma frequency synchronization by dendritic gabaergic synapses and gap junctions in a network of cortical interneurons. J Neurosci 21:5824-5831.

Traub RD, Whittington MA, Stanford IM, Jefferys JG (1996) A mechanism for generation of long-range synchronous fast oscillations in the cortex. Nature 383:621-624.

Traub RD, Kopell N, Bibbig A, Buhl EH, LeBeau FE, Whittington MA (2001) Gap junctions between interneuron dendrites can enhance synchrony of gamma oscillations in distributed networks. J Neurosci 21:9478-9486.

Tseng KY, O’Donnell P (2007) Dopamine modulation of prefrontal cortical interneurons changes during adolescence. Cereb Cortex 17:1235-1240.

Tseng KY, Lewis BL, Lipska BK, O’Donnell P (2007) Post-pubertal disruption of medial prefrontal cortical dopamine-glutamate interactions in a developmental animal model of schizophrenia. Biol Psychiatry 62:730-738.

Tseng KY, Lewis BL, Hashimoto T, Sesack SR, Kloc M, Lewis DA, O’Donnell $\mathrm{P}$ (2008) A neonatal ventral hippocampal lesion causes functional deficits in adult prefrontal cortical interneurons. J Neurosci 28:12691-12699.

Winterer G, Egan MF, Kolachana BS, Goldberg TE, Coppola R, Weinberger DR (2006) Prefrontal electrophysiologic "noise" and catechol-Omethyltransferase genotype in schizophrenia. Biol Psychiatry 60:578-584. 